\title{
Elucidating the role of fluorine content on gas sorption properties of fluorinated polyimides - Supporting Information
}

Albert X. Wu ${ }^{1}$, James A. Drayton ${ }^{1}$, Katherine Mizrahi Rodriguez ${ }^{2}$, Francesco M. Benedetti ${ }^{1}$, Qihui Qian ${ }^{1}$, Sharon Lin $^{1}$, and Zachary P. Smith ${ }^{1} *$

*Corresponding author: zpsmith@mit.edu

${ }^{1}$ Department of Chemical Engineering, Massachusetts Institute of Technology, Cambridge, MA 02139

${ }^{2}$ Department of Materials Science and Engineering, Massachusetts Institute of Technology, Cambridge, MA 02139 

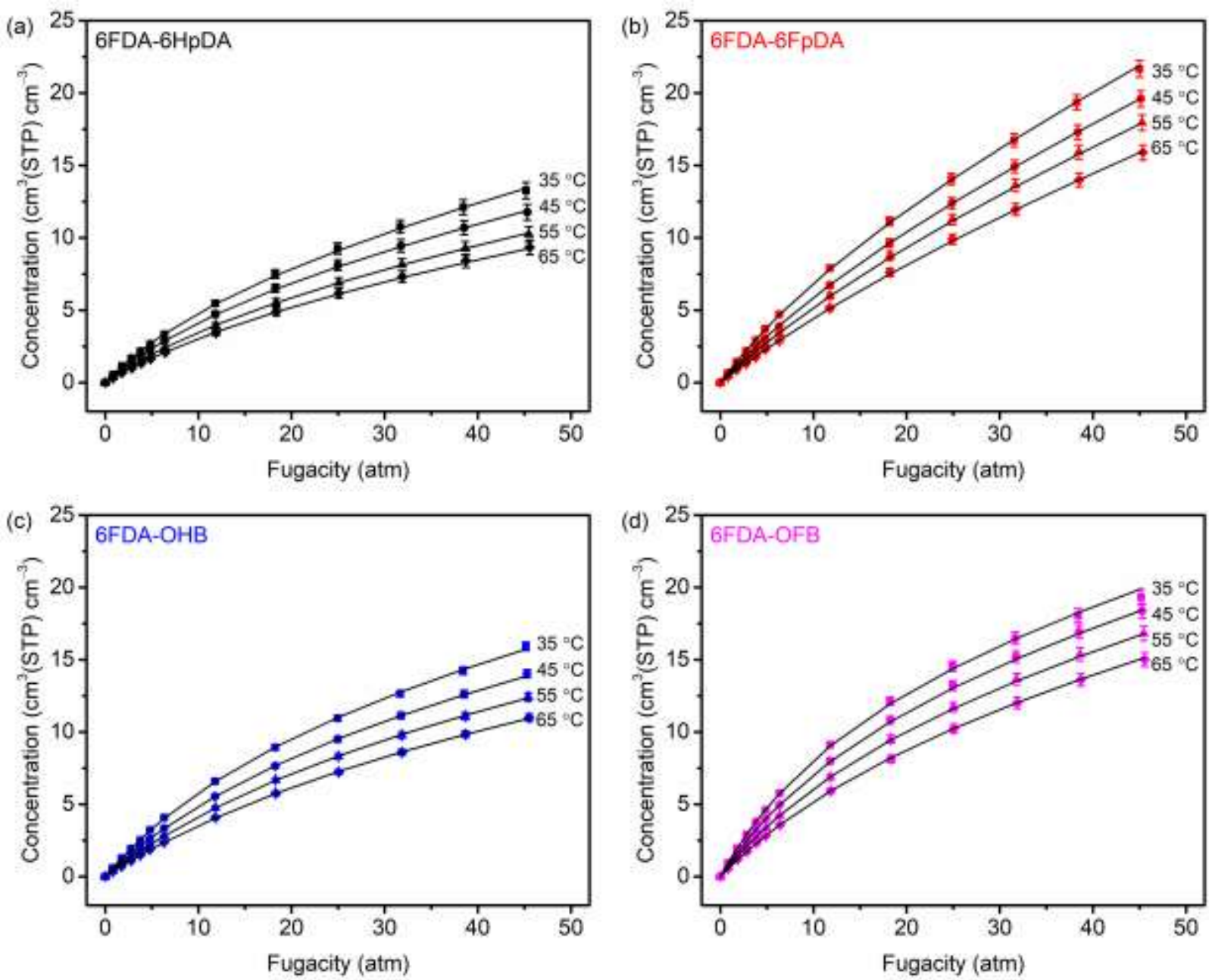

Figure S1: $\mathrm{N}_{2}$ isotherms for (a) 6FDA-6HpDA, (b) 6FDA-6FpDA, (c) 6FDA-OHB, and (d) 6FDA-OFB at $35{ }^{\circ} \mathrm{C}$ (squares), $45{ }^{\circ} \mathrm{C}$ (circles), $55{ }^{\circ} \mathrm{C}$ (triangles), and $65{ }^{\circ} \mathrm{C}$ (diamonds). Individual points indicate experimental data and lines indicate the dual-mode model fits. 

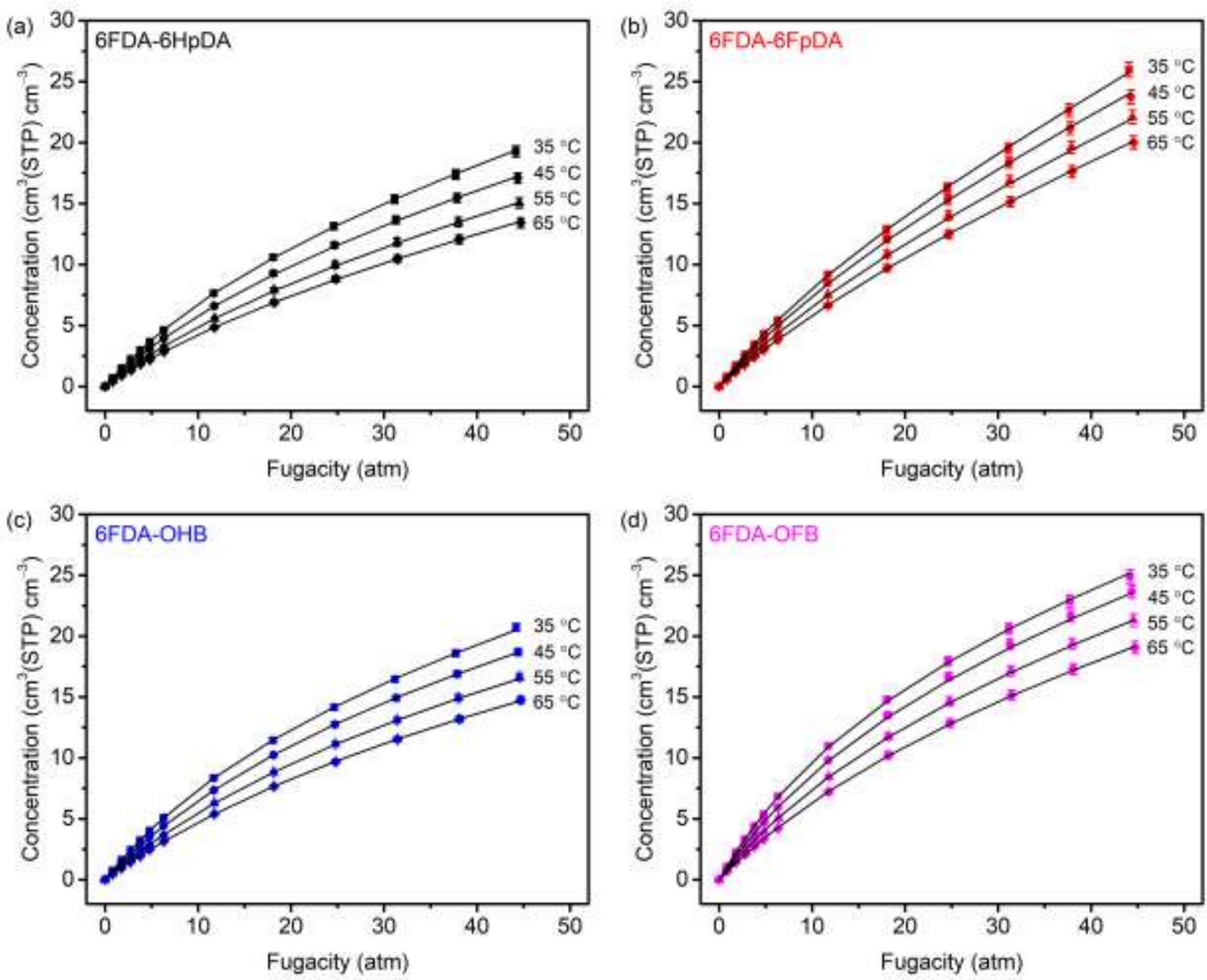

Figure S2: $\mathrm{O}_{2}$ isotherms for (a) 6FDA-6HpDA, (b) 6FDA-6FpDA, (c) 6FDA-OHB, and (d) 6FDA-OFB at $35{ }^{\circ} \mathrm{C}$ (squares), $45{ }^{\circ} \mathrm{C}$ (circles), $55{ }^{\circ} \mathrm{C}$ (triangles), and $65{ }^{\circ} \mathrm{C}$ (diamonds). Individual points indicate experimental data and lines indicate the dual-mode model fits. 

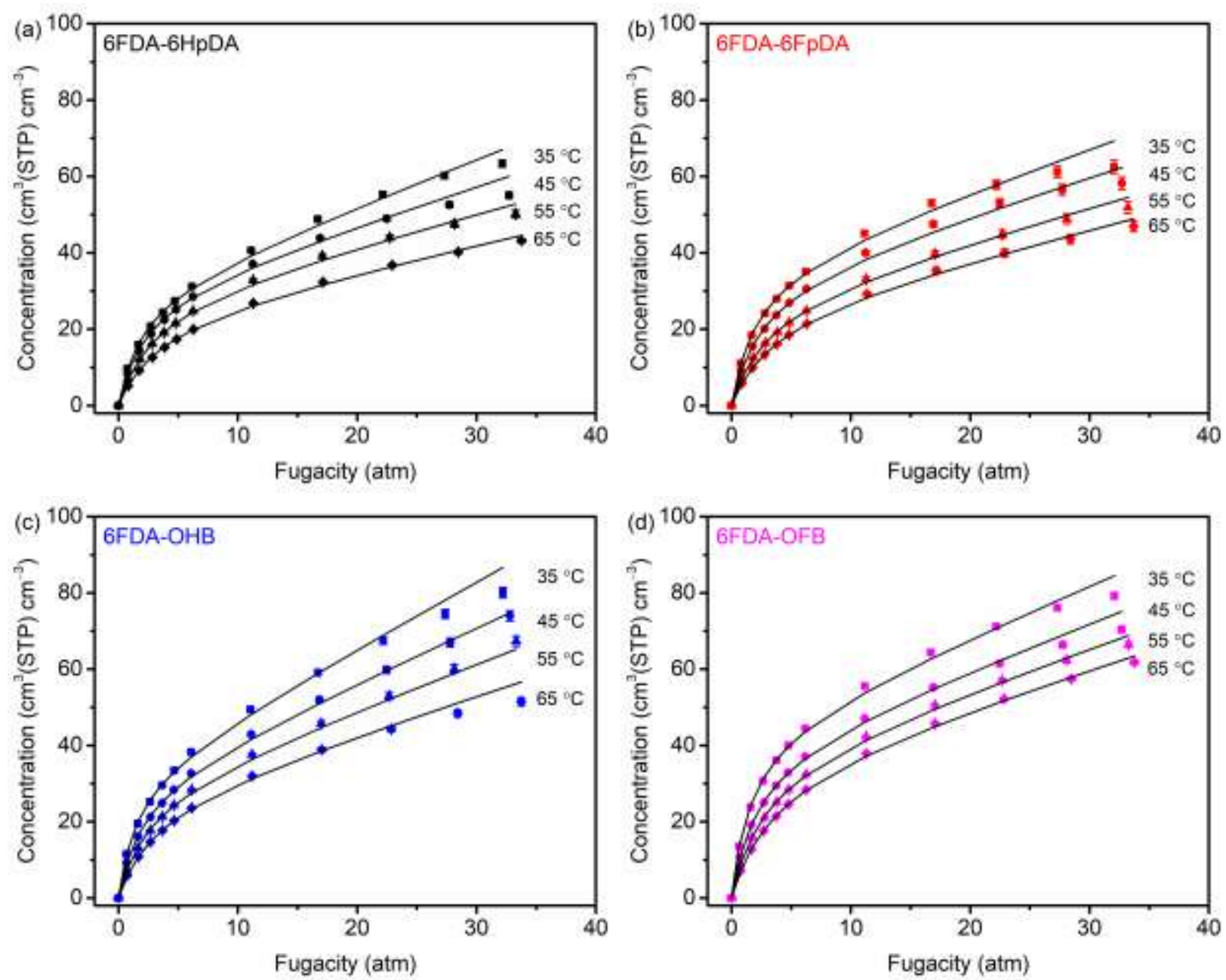

Figure S3: $\mathrm{CO}_{2}$ isotherms for (a) 6FDA-6HpDA, (b) 6FDA-6FpDA, (c) 6FDA-OHB, and (d) 6FDA-OFB at $35{ }^{\circ} \mathrm{C}$ (squares), $45{ }^{\circ} \mathrm{C}$ (circles), $55{ }^{\circ} \mathrm{C}$ (triangles), and $65{ }^{\circ} \mathrm{C}$ (diamonds). Individual points indicate experimental data and lines indicate the dual-mode model fits. 

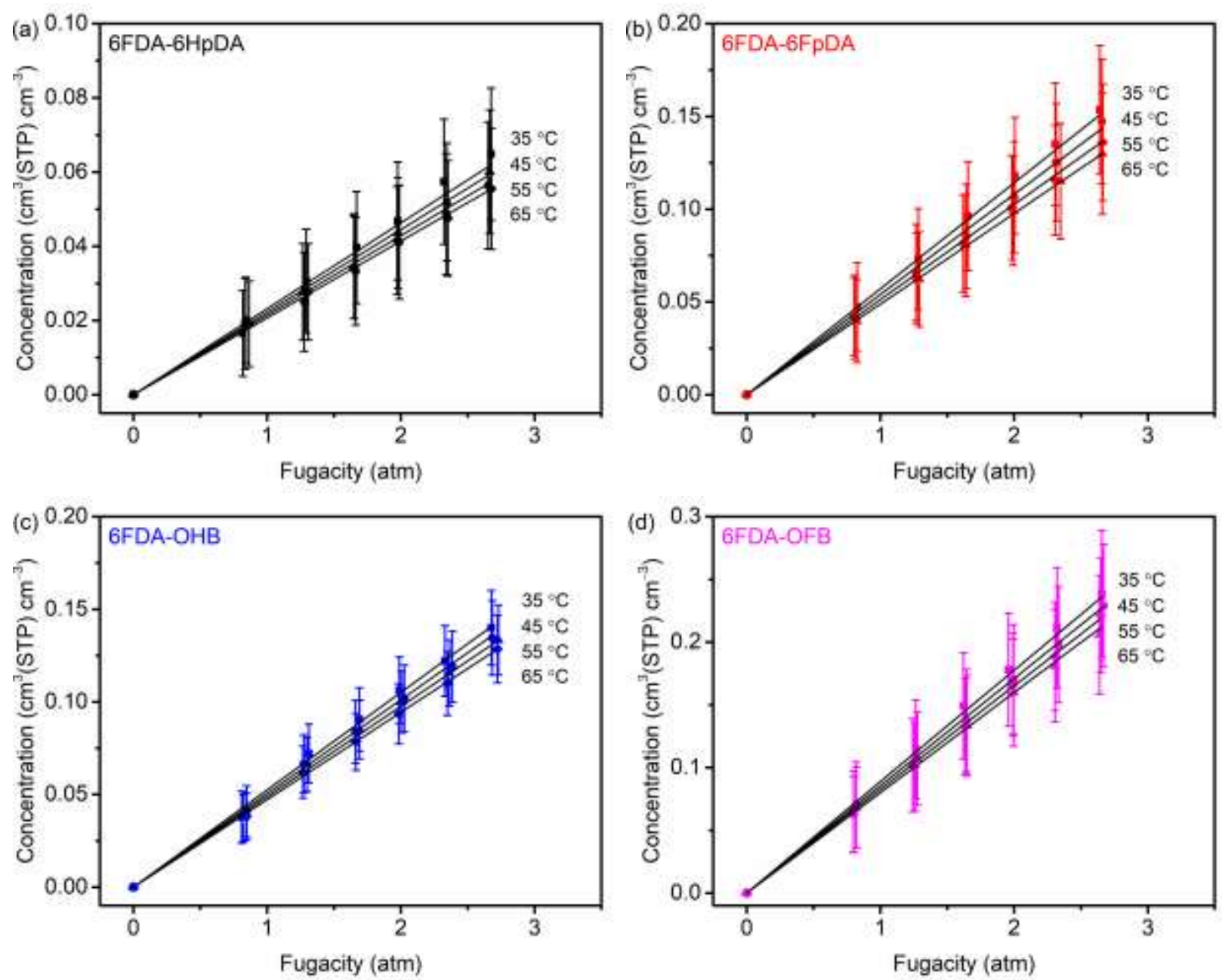

Figure S4: He isotherms for (a) 6FDA-6HpDA, (b) 6FDA-6FpDA, (c) 6FDA-OHB, (d) 6FDAOFB at $35{ }^{\circ} \mathrm{C}$ (squares), $45{ }^{\circ} \mathrm{C}$ (circles), $55{ }^{\circ} \mathrm{C}$ (triangles), and $65{ }^{\circ} \mathrm{C}$ (diamonds). Individual points indicate experimental data and lines indicate the constrained linear fits. 

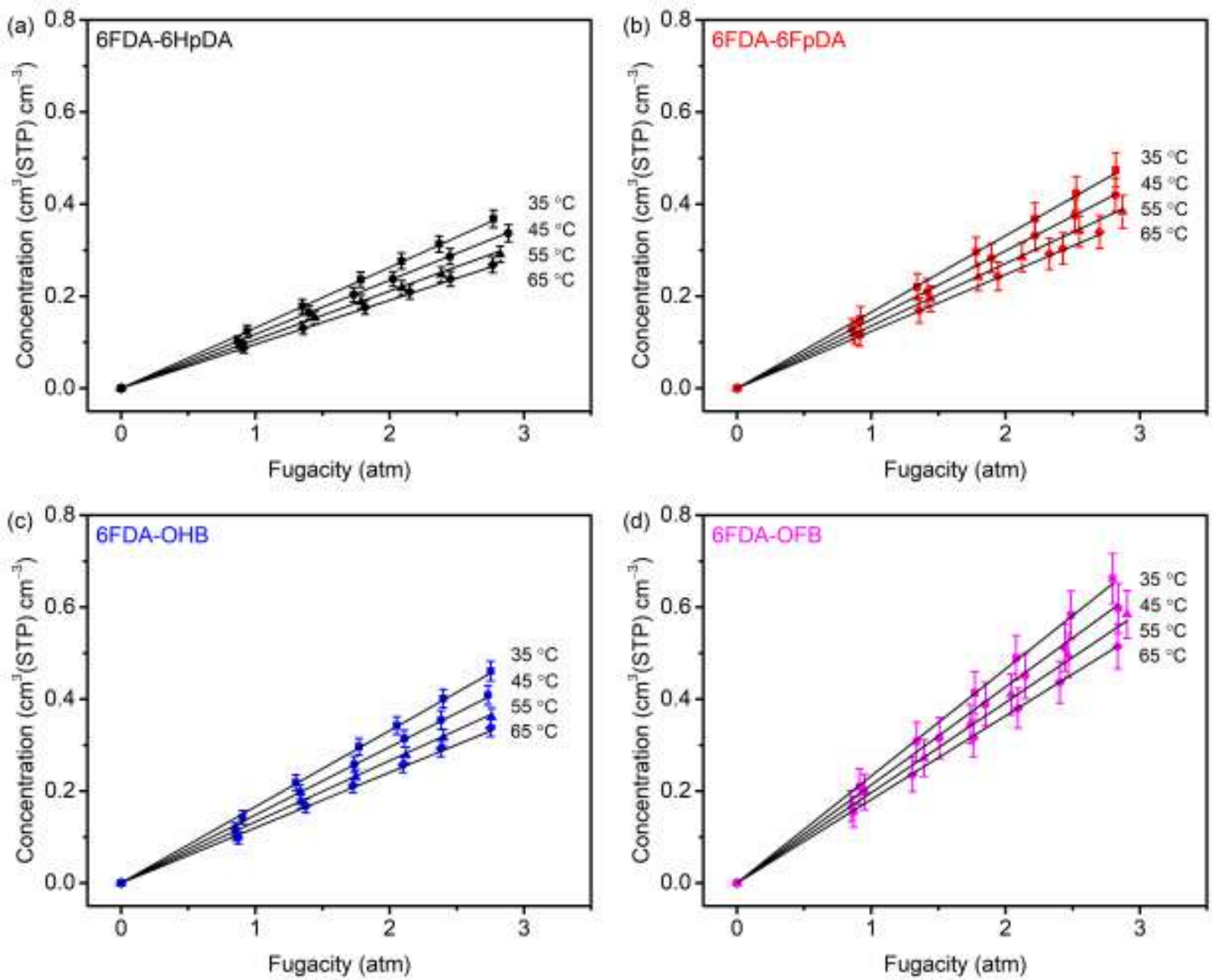

Figure S5: $\mathrm{H}_{2}$ isotherms for (a) 6FDA-6HpDA, (b) 6FDA-6FpDA, (c) 6FDA-OHB, (d) 6FDAOFB at $35{ }^{\circ} \mathrm{C}$ (squares), $45{ }^{\circ} \mathrm{C}$ (circles), $55{ }^{\circ} \mathrm{C}$ (triangles), and $65{ }^{\circ} \mathrm{C}$ (diamonds). Individual points indicate experimental data and lines indicate the constrained linear fits. 
Table S1: Summary of nonlinear optimization parameters and their upper and lower bound constraints. It should be noted that $C_{H}^{\prime}$ for $35{ }^{\circ} \mathrm{C}, 45{ }^{\circ} \mathrm{C}, 55{ }^{\circ} \mathrm{C}$, and $65{ }^{\circ} \mathrm{C}$ are each independent parameters, only constrained by relative order of magnitude as mentioned in the main text. An upper bound of 80 for $C_{H}^{\prime}$ values was chosen after internal refinement.

\begin{tabular}{|l|l|l|}
\hline Parameter & $\begin{array}{l}\text { Constraint } \\
{[\text { Lower bound, Upper bound }]}\end{array}$ & $\begin{array}{l}\text { Initial guess range } \\
{[\text { Lower bound, upper bound }]}\end{array}$ \\
\hline$k_{D, 0}\left(\mathrm{~cm}^{3}(\mathrm{STP}) \mathrm{cm}^{-3} \mathrm{~atm}^{-1}\right)$ & {$[0, \infty]$} & {$[0.1,1]$} \\
\hline$\Delta H_{D}\left(\mathrm{~kJ} \mathrm{~mol}^{-1}\right)$ & {$[-50,0]$} & {$[-10,-20]$} \\
\hline$b_{0}\left(\mathrm{~atm}^{-1}\right)$ & {$[0, \infty]$} & {$[0.001,0.1]$} \\
\hline$\Delta H_{b}\left(\mathrm{~kJ} \mathrm{~mol}^{-1}\right)$ & {$[-50,0]$} & {$[-10,-20]$} \\
\hline$C_{H}^{\prime}\left(\mathrm{cm}^{3}(\mathrm{STP}) \mathrm{cm}^{-3}\right)$ & {$[0,80]$} & {$[0,50]$} \\
\hline
\end{tabular}


Table S2: LFER parameters for the four polyimides. See Equations 12 and 13 in the main text for the corresponding units.

\begin{tabular}{|c|c|c|c|c|c|c|}
\hline 6FDA-6HpDA & $\mathrm{He}$ & $\mathrm{H}_{2}$ & $\mathrm{~N}_{2}$ & $\mathrm{O}_{2}$ & $\mathrm{CH}_{4}$ & $\mathrm{CO}_{2}$ \\
\hline$\alpha_{D}$ & 2.676 & 2.663 & $2.592 \pm 0.002$ & $2.576 \pm 0.001$ & $2.6323 \pm 0.0004$ & $2.6406 \pm 0.0002$ \\
\hline$\beta_{D}$ & 10.219 & 5.766 & $4.98 \pm 0.02$ & $3.96 \pm 0.01$ & $3.430 \pm 0.002$ & $0.0501 \pm 0.0008$ \\
\hline$\alpha_{b}$ & - & - & $2.632 \pm 0.003$ & $2.609 \pm 0.002$ & $2.6346 \pm 0.0004$ & $2.6734 \pm 0.0001$ \\
\hline$\beta_{b}$ & - & - & $7.84 \pm 0.03$ & $8.26 \pm 0.01$ & $5.486 \pm 0.003$ & $2.467 \pm 0.001$ \\
\hline 6FDA-6FpDA & $\mathrm{He}$ & $\mathrm{H}_{2}$ & $\mathrm{~N}_{2}$ & $\mathrm{O}_{2}$ & $\mathrm{CH}_{4}$ & $\mathrm{CO}_{2}$ \\
\hline$\alpha_{D}$ & 2.678 & 2.665 & $2.677 \pm 0.004$ & $2.739 \pm 0.002$ & $2.5907 \pm 0.0008$ & $\begin{array}{l}2.6488 \pm 0.0004 \\
\end{array}$ \\
\hline$\beta_{D}$ & 7.875 & 5.130 & $3.63 \pm 0.02$ & $3.01 \pm 0.01$ & $1.692 \pm 0.004$ & $0.150 \pm 0.001$ \\
\hline$\alpha_{b}$ & - & & $2.65 \pm 0.02$ & $2.58 \pm 0.01$ & $2.565 \pm 0.002$ & $2.6410 \pm 0.0002$ \\
\hline$\beta_{b}$ & - & - & $8.79 \pm 0.2$ & $7.7 \quad \pm 0.1$ & $4.71 \quad \pm 0.02$ & $2.373 \pm 0.001$ \\
\hline 6FDA-OHB & $\mathrm{He}$ & $\mathrm{H}_{2}$ & $\mathrm{~N}_{2}$ & $\mathrm{O}_{2}$ & $\mathrm{CH}_{4}$ & $\mathrm{CO}_{2}$ \\
\hline$\alpha_{D}$ & 2.681 & 2.666 & $2.575 \pm 0.002$ & $\overline{2.564 \pm 0.002}$ & $2.6272 \pm 0.0003$ & $2.6439 \pm 0.0005$ \\
\hline$\beta_{D}$ & 8.040 & 5.169 & $5.49 \pm 0.01$ & $4.45 \pm 0.01$ & $2.984 \pm 0.002$ & $-0.897 \pm 0.003$ \\
\hline$\alpha_{b}$ & - & & $2.570 \pm 0.002$ & $2.560 \pm 0.002$ & $2.6292 \pm 0.0004$ & $2.6469 \pm 0.0008$ \\
\hline$\beta_{b}$ & - & - & $8.27 \pm 0.02$ & $8.27 \pm 0.02$ & $5.112 \pm 0.004$ & $1.716 \pm 0.007$ \\
\hline 6FDA-OFB & $\mathrm{He}$ & $\mathrm{H}_{2}$ & $\mathrm{~N}_{2}$ & $\mathrm{O}_{2}$ & $\mathrm{CH}_{4}$ & $\mathrm{CO}_{2}$ \\
\hline$\alpha_{D}$ & 2.684 & 2.670 & $2.539 \pm 0.001$ & $2.543 \pm 0.001$ & $2.5978 \pm 0.0004$ & $2.6454 \pm 0.0003$ \\
\hline$\beta_{D}$ & 6.644 & 4.192 & $4.747 \pm 0.009$ & $3.886 \pm 0.008$ & $3.360 \pm 0.003$ & $-0.402 \pm 0.002$ \\
\hline$\alpha_{b}$ & - & - & $2.529 \pm 0.002$ & $2.527 \pm 0.002$ & $2.5950 \pm 0.0006$ & $2.6436 \pm 0.0004$ \\
\hline$\beta_{b}$ & - & - & $7.13 \pm 0.02$ & $7.47 \pm 0.02$ & $5.295 \pm 0.005$ & $1.982 \pm 0.004$ \\
\hline
\end{tabular}


Table S3: Best-fit parameters for the four polyimides. $\Delta H_{S_{\infty}}, \Delta H_{D}$, and $\Delta H_{b}$ are in units of kJ $\mathrm{mol}^{-1}$ and all $C_{H}^{\prime}$ values are in units of $\mathrm{cm}^{3}(\mathrm{STP}) \mathrm{cm}^{-3}$. $\Delta H_{S_{\infty}}$ values for $\mathrm{He}$ and $\mathrm{H}_{2}$ were obtained via constrained linear optimization. $\Delta H_{S_{\infty}}$ values for the other four gases were obtained using the calculated $S_{\infty}$ values in Tables $\mathrm{S} 4-\mathrm{S} 7$ below.

\begin{tabular}{|c|c|c|c|c|c|c|}
\hline 6FDA-6HpDA & $\mathrm{He}$ & $\mathrm{H}_{2}$ & $\mathrm{~N}_{2}$ & $\mathrm{O}_{2}$ & $\mathrm{CH}_{4}$ & $\mathrm{CO}_{2}$ \\
\hline$\Delta H_{S_{\infty}}$ & $-3 \pm 4$ & $-9 \pm 1$ & $-16.3 \pm 0.2$ & $-16.0 \pm 0.2$ & $-20.2 \pm 0.2$ & $-23.9 \pm 0.9$ \\
\hline$\Delta H_{D}$ & - & - & $-11.9 \pm 0.6$ & $-10.8 \pm 0.3$ & $-11.2 \pm 0.2$ & $-16.91 \pm 0.03$ \\
\hline$\Delta H_{b}$ & - & - & $-11.3 \pm 0.5$ & $-11.0 \pm 0.2$ & $-16.8 \pm 0.2$ & $-18.48 \pm 0.05$ \\
\hline$C_{H, 35^{\circ} \mathrm{C}}^{\prime}$ & - & - & $8.9 \pm 0.1$ & $14.5 \pm 0.1$ & $15.59 \pm 0.07$ & $30.25 \pm 0.05$ \\
\hline$C_{H, 45^{\circ} \mathrm{C}}^{\prime}$ & - & - & $8.4 \pm 0.1$ & $13.6 \pm 0.2$ & $15.03 \pm 0.08$ & $30.25 \pm 0.05$ \\
\hline$C_{H, 55^{\circ} \mathrm{C}}^{\prime}$ & - & - & $7.6 \pm 0.2$ & $12.2 \pm 0.2$ & $13.99 \pm 0.09$ & $28.49 \pm 0.08$ \\
\hline$C_{H, 65^{\circ} \mathrm{C}}^{\prime}$ & - & - & $7.2 \pm 0.2$ & $11.5 \pm 0.2$ & $13.26 \pm 0.09$ & $24.40 \pm 0.05$ \\
\hline 6FDA-6FpDA & $\mathrm{He}$ & $\mathrm{H}_{2}$ & $\mathrm{~N}_{2}$ & $\mathrm{O}_{2}$ & $\mathrm{CH}_{4}$ & $\mathrm{CO}_{2}$ \\
\hline$\Delta H_{S_{\infty}}$ & $-5 \pm 4$ & $-8 \pm 2$ & $-15.7 \pm 0.2$ & $-12.2 \pm 0.3$ & $-19.2 \pm 0.2$ & $-25.6 \pm 0.3$ \\
\hline$\Delta H_{D}$ & - & - & $\begin{array}{l}-7.6 \pm 0.8 \\
\end{array}$ & $-6 \pm 1$ & $\begin{array}{l}-5.6 \pm 0.6 \\
\end{array}$ & $-10.1 \pm 0.1$ \\
\hline$\Delta H_{b}$ & - & - & $-16.6 \pm 0.2$ & $-11 \pm 1$ & $-14.3 \pm 0.5$ & $-16.2 \pm 0.1$ \\
\hline$C_{H, 35^{\circ} \mathrm{C}}^{\prime}$ & - & - & $12.9 \pm 0.6$ & $12.0 \pm 0.6$ & $14.8 \pm 0.3$ & $37.0 \pm 0.2$ \\
\hline$C_{H, 45^{\circ} \mathrm{C}}^{\prime}$ & - & - & $12.1 \pm 0.7$ & $12.0 \pm 0.6$ & $13.7 \pm 0.3$ & $33.6 \pm 0.2$ \\
\hline$C_{H, 55^{\circ} \mathrm{C}}^{\prime}$ & - & - & $11.9 \pm 0.8$ & $11.0 \pm 0.7$ & $12.3 \pm 0.3$ & $28.8 \pm 0.2$ \\
\hline$C_{H, 65^{\circ} \mathrm{C}}^{\prime}$ & - & - & $10.9 \pm 0.9$ & $10.1 \pm 0.8$ & $10.8 \pm 0.3$ & $25.8 \pm 0.2$ \\
\hline
\end{tabular}




\begin{tabular}{|c|c|c|c|c|c|c|}
\hline 6FDA-OHB & $\mathrm{He}$ & $\mathrm{H}_{2}$ & $\mathrm{~N}_{2}$ & $\mathrm{O}_{2}$ & $\mathrm{CH}_{4}$ & $\mathrm{CO}_{2}$ \\
\hline$\Delta H_{S_{\infty}}$ & $-3 \pm 2$ & 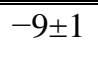 & 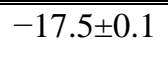 & $-16.1 \pm 0.2$ & 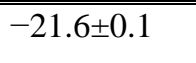 & $\begin{array}{l}-26.8 \pm 0.1 \\
\end{array}$ \\
\hline$\Delta H_{D}$ & - & - & 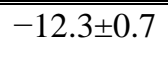 & $-12.5 \pm 0.7$ & $-13.1 \pm 0.3$ & $-16.43 \pm 0.09$ \\
\hline$\Delta H_{b}$ & - & - & $-15.1 \pm 0.5$ & $-14.5 \pm 0.4$ & $-19.4 \pm 0.2$ & $-21.2 \pm 0.2$ \\
\hline$C_{H, 35^{\circ} \mathrm{C}}^{\prime}$ & - & - & $15.8 \pm 0.2$ & $16.6 \pm 0.3$ & $16.7 \pm 0.1$ & $32.9 \pm 0.2$ \\
\hline$C_{H, 45^{\circ} \mathrm{C}}^{\prime}$ & - & - & $15.0 \pm 0.3$ & $16.6 \pm 0.3$ & $16.1 \pm 0.1$ & $30.5 \pm 0.2$ \\
\hline$C_{H, 55^{\circ} \mathrm{C}}^{\prime}$ & - & - & $14.5 \pm 0.3$ & $15.8 \pm 0.3$ & $15.5 \pm 0.1$ & $28.5 \pm 0.2$ \\
\hline$C_{H, 65{ }^{\circ} \mathrm{C}}^{\prime}$ & - & - & $13.9 \pm 0.3$ & $15.1 \pm 0.4$ & $14.8 \pm 0.1$ & $26.0 \pm 0.2$ \\
\hline 6FDA-OFB & $\mathrm{He}$ & $\mathrm{H}_{2}$ & $\mathrm{~N}_{2}$ & $\mathrm{O}_{2}$ & $\overline{\mathrm{CH}_{4}}$ & $\mathrm{CO}_{2}$ \\
\hline$\Delta H_{S_{\infty}}$ & $-3 \pm 2$ & $-7 \pm 1$ & $-16.6 \pm 0.2$ & $-15.8 \pm 0.3$ & $-21.1 \pm 0.7$ & $-27.9 \pm 0.4$ \\
\hline$\Delta H_{D}$ & - & - & $-3.0 \pm 0.2$ & $-4.6 \pm 0.1$ & $\begin{array}{c}-0.17 \pm 0.02 \\
\end{array}$ & $-8.98 \pm 0.01$ \\
\hline$\Delta H_{b}$ & - & - & $-12.3 \pm 0.2$ & $-12.5 \pm 0.2$ & $-11.71 \pm 0.08$ & $-21.59 \pm 0.03$ \\
\hline$C_{H, 35^{\circ} \mathrm{C}}^{\prime}$ & - & - & $18.1 \pm 0.2$ & $23.0 \pm 0.2$ & $24.44 \pm 0.08$ & $44.94 \pm 0.03$ \\
\hline$C_{H, 45^{\circ} \mathrm{C}}^{\prime}$ & - & - & $17.2 \pm 0.1$ & $22.4 \pm 0.2$ & $22.28 \pm 0.07$ & $39.43 \pm 0.06$ \\
\hline$C_{H, 55^{\circ} \mathrm{C}}^{\prime}$ & - & - & $15.8 \pm 0.2$ & $20.6 \pm 0.2$ & $19.84 \pm 0.08$ & $36.65 \pm 0.06$ \\
\hline$C_{H, 65{ }^{\circ} \mathrm{C}}^{\prime}$ & - & - & $14.2 \pm 0.1$ & $18.7 \pm 0.2$ & $16.0 \pm 0.1$ & $34.49 \pm 0.05$ \\
\hline
\end{tabular}


Table S4: Calculated best-fit dual mode model parameters for 6FDA-6HpDA. $k_{D}$ values are in units of $\mathrm{cm}^{3}$ (STP) $\mathrm{cm}^{-3} \mathrm{~atm}^{-1}, b$ values are in units of $\mathrm{atm}^{-1}$, and $S_{\infty}$ values are in units of $\mathrm{cm}^{3}(\mathrm{STP}) \mathrm{cm}^{-3} \mathrm{~atm}^{-1}$.

\begin{tabular}{|c|c|c|c|c|c|c|}
\hline $35^{\circ} \mathrm{C}$ & $\mathrm{He}$ & $\mathrm{H}_{2}$ & $\mathrm{~N}_{2}$ & $\mathrm{O}_{2}$ & $\mathrm{CH}_{4}$ & $\mathrm{CO}_{2}$ \\
\hline$k_{D}$ & - & - & $0.154 \pm 0.001$ & $0.220 \pm 0.001$ & $0.3054 \pm 0.0008$ & $1.1943 \pm 0.0008$ \\
\hline$b$ & - & - & $0.0572 \pm 0.0008$ & $0.0456 \pm 0.0003$ & $0.1494 \pm 0.0004$ & $0.5368 \pm 0.0006$ \\
\hline$S_{\infty}$ & $0.023 \pm 0.001$ & $0.131 \pm 0.002$ & $0.67 \quad \pm 0.01$ & $0.883 \pm 0.008$ & $2.63 \pm 0.01$ & $17.43 \pm 0.03$ \\
\hline $45^{\circ} \mathrm{C}$ & $\mathrm{He}$ & $\mathrm{H}_{2}$ & $\mathrm{~N}_{2}$ & $\mathrm{O}_{2}$ & $\mathrm{CH}_{4}$ & $\mathrm{CO}_{2}$ \\
\hline$k_{D}$ & - & - & $0.133 \pm 0.0 .001$ & $0.193 \pm 0.0 .001$ & $0.2661 \pm 0.0003$ & $0.9706 \pm 0.0006$ \\
\hline$b$ & - & - & $0.0498 \pm 0.0006$ & $0.0399 \pm 0.0003$ & $0.1215 \pm 0.0002$ & $0.4279 \pm 0.0003$ \\
\hline$S_{\infty}$ & $0.0223 \pm 0.0003$ & $0.1174 \pm 0.0004$ & $0.552 \pm 0.009$ & $0.735 \pm 0.007$ & $2.09 \quad \pm 0.01$ & $13.91 \quad \pm 0.03$ \\
\hline $55^{\circ} \mathrm{C}$ & $\mathrm{He}$ & $\mathrm{H}_{2}$ & $\mathrm{~N}_{2}$ & $\mathrm{O}_{2}$ & $\mathrm{CH}_{4}$ & $\mathrm{CO}_{2}$ \\
\hline$k_{D}$ & - & - & $0.116 \pm 0.002$ & $0.174 \pm 0.001$ & $0.2339 \pm 0.0008$ & $0.7988 \pm 0.0005$ \\
\hline$b$ & - & - & $0.0437 \pm 0.0006$ & $0.0351 \pm 0.0003$ & $0.1001 \pm 0.0003$ & $0.3459 \pm 0.0003$ \\
\hline$S_{\infty}$ & $0.0214 \pm 0.0006$ & $0.106 \pm 0.001$ & $0.448 \pm 0.008$ & $0.600 \pm 0.006$ & $1.63 \pm 0.01$ & $10.65 \pm 0.03$ \\
\hline $65^{\circ} \mathrm{C}$ & $\mathrm{He}$ & $\mathrm{H}_{2}$ & $\mathrm{~N}_{2}$ & $\mathrm{O}_{2}$ & $\mathrm{CH}_{4}$ & $\mathrm{CO}_{2}$ \\
\hline$k_{D}$ & - & - & $0.102 \pm 0.002$ & $0.151 \pm 0.002$ & $0.207 \pm 0.001$ & $0.665 \pm 0.0006$ \\
\hline$b$ & - & - & $0.0386 \pm 0.0007$ & $0.0312 \pm 0.0003$ & $0.0834 \pm 0.0004$ & $0.2831 \pm 0.0003$ \\
\hline$S_{\infty}$ & $0.021 \pm 0.001$ & $0.096 \pm 0.002$ & $0.381 \pm 0.008$ & $0.51 \pm 0.006$ & $1.313 \pm 0.01$ & $7.57 \quad \pm 0.02$ \\
\hline
\end{tabular}


Table S5: Calculated best-fit dual mode model parameters for 6FDA-6FpDA. $k_{D}$ values are in units of $\mathrm{cm}^{3}$ (STP) $\mathrm{cm}^{-3} \mathrm{~atm}^{-1}, b$ values are in units of $\mathrm{atm}^{-1}$, and $S_{\infty}$ values are in units of $\mathrm{cm}^{3}(\mathrm{STP}) \mathrm{cm}^{-3} \mathrm{~atm}^{-1}$.

\begin{tabular}{|c|c|c|c|c|c|c|}
\hline $35^{\circ} \mathrm{C}$ & $\mathrm{He}$ & $\mathrm{H}_{2}$ & $\mathrm{~N}_{2}$ & $\mathrm{O}_{2}$ & $\mathrm{CH}_{4}$ & $\mathrm{CO}_{2}$ \\
\hline$k_{D}$ & - & - & $0.293 \pm 0.005$ & $0.39 \pm 0.01$ & $0.533 \pm 0.002$ & $1.075 \pm 0.002$ \\
\hline$b$ & - & - & $0.044 \pm 0.005$ & $0.053 \pm 0.003$ & $0.161 \pm 0.001$ & $0.4919 \pm 0.0007$ \\
\hline$S_{\infty}$ & $0.057 \pm 0.004$ & $0.166 \pm 0.004$ & $0.86 \pm 0.07$ & $1.03 \pm 0.04$ & $2.91 \pm 0.05$ & $19.3 \quad \pm 0.1$ \\
\hline $45^{\circ} \mathrm{C}$ & $\mathrm{He}$ & $\mathrm{H}_{2}$ & $\mathrm{~N}_{2}$ & $\mathrm{O}_{2}$ & $\mathrm{CH}_{4}$ & $\mathrm{CO}_{2}$ \\
\hline$k_{D}$ & - & - & $0.267 \pm 0.003$ & $0.362 \pm 0.006$ & $0.498 \pm 0.002$ & $0.9499 \pm 0.0008$ \\
\hline$b$ & - & - & $0.036 \pm 0.004$ & $0.046 \pm 0.002$ & $0.135 \pm 0.001$ & $0.4034 \pm 0.0003$ \\
\hline$S_{\infty}$ & $0.054 \pm 0.001$ & $0.1494 \pm 0.0007$ & $0.70 \pm 0.05$ & $0.91 \pm 0.04$ & $2.34 \pm 0.04$ & $14.51 \quad \pm 0.09$ \\
\hline $55^{\circ} \mathrm{C}$ & $\mathrm{He}$ & $\mathrm{H}_{2}$ & $\mathrm{~N}_{2}$ & $\mathrm{O}_{2}$ & $\mathrm{CH}_{4}$ & $\mathrm{CO}_{2}$ \\
\hline$k_{D}$ & - & - & $0.244 \pm 0.003$ & $0.336 \pm 0.002$ & $0.467 \pm 0.005$ & $0.846 \pm 0.001$ \\
\hline$b$ & - & - & $0.030 \pm 0.003$ & $0.040 \pm 0.002$ & $0.114 \pm 0.002$ & $0.3349 \pm 0.0005$ \\
\hline$S_{\infty}$ & $0.051 \pm 0.001$ & $0.136 \pm 0.002$ & $0.60 \pm 0.05$ & $0.78 \pm 0.04$ & $1.87 \pm 0.04$ & $10.49 \quad \pm 0.08$ \\
\hline $65^{\circ} \mathrm{C}$ & $\mathrm{He}$ & $\mathrm{H}_{2}$ & $\mathrm{~N}_{2}$ & $\mathrm{O}_{2}$ & $\mathrm{CH}_{4}$ & $\mathrm{CO}_{2}$ \\
\hline$k_{D}$ & - & - & $0.225 \pm 0.004$ & $0.314 \pm 0.004$ & $0.439 \pm 0.008$ & $0.758 \pm 0.002$ \\
\hline$b$ & - & - & $0.025 \pm 0.003$ & $0.036 \pm 0.002$ & $0.098 \pm 0.002$ & $0.2812 \pm 0.0007$ \\
\hline$S_{\infty}$ & $0.049 \pm 0.004$ & $0.124 \pm 0.004$ & $0.50 \pm 0.04$ & $0.67 \pm 0.04$ & $1.50 \pm 0.04$ & $8.01 \quad \pm 0.07$ \\
\hline
\end{tabular}


Table S6: Calculated best-fit dual mode model parameters for 6FDA-OHB. $k_{D}$ values are in units of $\mathrm{cm}^{3}$ (STP) $\mathrm{cm}^{-3} \mathrm{~atm}^{-1}, b$ values are in units of $\mathrm{atm}^{-1}$, and $S_{\infty}$ values are in units of $\mathrm{cm}^{3}(\mathrm{STP}) \mathrm{cm}^{-3} \mathrm{~atm}^{-1}$.

\begin{tabular}{|c|c|c|c|c|c|c|}
\hline $35^{\circ} \mathrm{C}$ & $\mathrm{He}$ & $\mathrm{H}_{2}$ & $\mathrm{~N}_{2}$ & $\mathrm{O}_{2}$ & $\mathrm{CH}_{4}$ & $\mathrm{CO}_{2}$ \\
\hline$k_{D}$ & - & - & $0.1216 \pm 0.0009$ & $0.224 \pm 0.001$ & $0.365 \pm 0.001$ & $1.713 \pm 0.003$ \\
\hline$b$ & - & - & $0.0408 \pm 0.0004$ & $0.0449 \pm 0.0005$ & $0.1737 \pm 0.0005$ & $0.682 \pm 0.003$ \\
\hline$S_{\infty}$ & $0.053 \pm 0.002$ & $0.166 \pm 0.003$ & $0.77 \quad \pm 0.01$ & $0.97 \quad \pm 0.01$ & $3.27 \quad \pm 0.02$ & $24.2 \pm 0.2$ \\
\hline $45^{\circ} \mathrm{C}$ & $\mathrm{He}$ & $\mathrm{H}_{2}$ & $\mathrm{~N}_{2}$ & $\mathrm{O}_{2}$ & $\mathrm{CH}_{4}$ & $\mathrm{CO}_{2}$ \\
\hline$k_{D}$ & - & - & $0.105 \pm 0.001$ & $0.192 \pm 0.002$ & $0.3106 \pm 0.0004$ & $1.400 \pm 0.002$ \\
\hline$b$ & - & & $0.0339 \pm 0.0004$ & $0.0375 \pm 0.0004$ & $0.1369 \pm 0.0003$ & $0.526 \pm 0.002$ \\
\hline$S_{\infty}$ & $0.0506 \pm 0.0006$ & $0.148 \pm 0.002$ & $0.62 \pm 0.01$ & $0.81 \quad \pm 0.01$ & $2.52 \pm 0.01$ & $\begin{array}{ll}17.3 \quad \pm 0.1 \\
\end{array}$ \\
\hline $55^{\circ} \mathrm{C}$ & $\mathrm{He}$ & $\mathrm{H}_{2}$ & $\mathrm{~N}_{2}$ & $\mathrm{O}_{2}$ & $\mathrm{CH}_{4}$ & $\mathrm{CO}_{2}$ \\
\hline$k_{D}$ & - & - & $\begin{array}{c}0.092 \pm 0.001 \\
\end{array}$ & $\begin{array}{c}0.167 \pm 0.002 \\
\end{array}$ & $\begin{array}{c}0.267 \pm 0.001 \\
\end{array}$ & $1.159 \pm 0.002$ \\
\hline$b$ & - & - & $0.0285 \pm 0.0004$ & $0.0317 \pm 0.0004$ & $0.1094 \pm 0.0004$ & $0.412 \pm 0.002$ \\
\hline$S_{\infty}$ & $0.0488 \pm 0.0008$ & $0.133 \pm 0.002$ & $0.51 \quad \pm 0.01$ & $0.67 \quad \pm 0.01$ & $1.97 \quad \pm 0.01$ & $12.9 \quad \pm 0.1$ \\
\hline $65^{\circ} \mathrm{C}$ & $\mathrm{He}$ & $\mathrm{H}_{2}$ & $\mathrm{~N}_{2}$ & $\mathrm{O}_{2}$ & $\mathrm{CH}_{4}$ & $\mathrm{CO}_{2}$ \\
\hline$k_{D}$ & - & - & $0.080 \pm 0.002$ & $0.145 \pm 0.003$ & $0.232 \pm 0.002$ & $0.970 \pm 0.003$ \\
\hline$b$ & - & - & $0.0243 \pm 0.0005$ & $0.0271 \pm 0.0004$ & $0.0887 \pm 0.0005$ & $0.328 \pm 0.002$ \\
\hline$S_{\infty}$ & $0.047 \pm 0.002$ & $0.120 \pm 0.003$ & $0.42 \quad \pm 0.01$ & $\begin{array}{ll}0.56 \quad \pm 0.01 \\
\end{array}$ & $1.55 \quad \pm 0.01$ & $9.50 \pm 0.09$ \\
\hline
\end{tabular}


Table S7: Calculated best-fit dual mode model parameters for 6FDA-OFB. $k_{D}$ values are in units of $\mathrm{cm}^{3}$ (STP) $\mathrm{cm}^{-3} \mathrm{~atm}^{-1}, b$ values are in units of $\mathrm{atm}^{-1}$, and $S_{\infty}$ values are in units of $\mathrm{cm}^{3}(\mathrm{STP}) \mathrm{cm}^{-3} \mathrm{~atm}^{-1}$.

\begin{tabular}{|c|c|c|c|c|c|c|}
\hline $35^{\circ} \mathrm{C}$ & $\mathrm{He}$ & $\mathrm{H}_{2}$ & $\mathrm{~N}_{2}$ & $\mathrm{O}_{2}$ & $\mathrm{CH}_{4}$ & $\mathrm{CO}_{2}$ \\
\hline$k_{D}$ & - & - & $0.1526 \pm 0.0006$ & $0.2141 \pm 0.0008$ & $0.2743 \pm 0.0003$ & $1.3001 \pm 0.0009$ \\
\hline$b$ & - & - & $0.0561 \pm 0.0005$ & $0.0488 \pm 0.0005$ & $0.1379 \pm 0.0003$ & $0.613 \pm 0.001$ \\
\hline$S_{\infty}$ & $0.089 \pm 0.004$ & $0.233 \pm 0.005$ & $1.17 \quad \pm 0.02$ & $1.34 \quad \pm 0.01$ & $3.63 \quad \pm 0.01$ & $28.85 \quad \pm 0.05$ \\
\hline $45^{\circ} \mathrm{C}$ & $\mathrm{He}$ & $\mathrm{H}_{2}$ & $\mathrm{~N}_{2}$ & $\mathrm{O}_{2}$ & $\mathrm{CH}_{4}$ & $\mathrm{CO}_{2}$ \\
\hline$k_{D}$ & - & - & $0.1470 \pm 0.0007$ & $0.2024 \pm 0.0008$ & $0.2743 \pm 0.0003$ & $1.1646 \pm 0.0008$ \\
\hline$b$ & . & - & $0.0482 \pm 0.0005$ & $0.0419 \pm 0.0004$ & $0.1191 \pm 0.0003$ & $0.4704 \pm 0.0009$ \\
\hline$S_{\infty}$ & $0.086 \pm 0.001$ & $0.213 \pm 0.001$ & $0.97 \quad \pm 0.01$ & $1.14 \quad \pm 0.01$ & $2.92 \pm 0.01$ & $19.72 \pm 0.04$ \\
\hline $55^{\circ} \mathrm{C}$ & $\mathrm{He}$ & $\mathrm{H}_{2}$ & $\mathrm{~N}_{2}$ & $\mathrm{O}_{2}$ & $\mathrm{CH}_{4}$ & $\mathrm{CO}_{2}$ \\
\hline$k_{D}$ & - & - & $0.1419 \pm 0.0008$ & $0.192 \pm 0.001$ & $0.2743 \pm 0.0003$ & $1.0501 \pm 0.0008$ \\
\hline$b$ & - & - & $0.0418 \pm 0.0005$ & $0.0363 \pm 0.0004$ & $0.1039 \pm 0.0003$ & $0.3668 \pm 0.0007$ \\
\hline$S_{\infty}$ & $0.083 \pm 0.001$ & $0.196 \pm 0.002$ & $0.80 \quad \pm 0.01$ & $0.94 \quad \pm 0.01$ & $2.33 \quad \pm 0.01$ & $14.49 \quad \pm 0.03$ \\
\hline $65^{\circ} \mathrm{C}$ & $\mathrm{He}$ & $\mathrm{H}_{2}$ & $\mathrm{~N}_{2}$ & $\mathrm{O}_{2}$ & $\mathrm{CH}_{4}$ & $\mathrm{CO}_{2}$ \\
\hline$k_{D}$ & - & - & $0.137 \pm 0.001$ & $0.183 \pm 0.001$ & $0.2743 \pm 0.0003$ & $0.9528 \pm 0.0007$ \\
\hline$b$ & - & - & $0.0366 \pm 0.0005$ & $0.0317 \pm 0.0004$ & $0.0913 \pm 0.0003$ & $0.2903 \pm 0.0006$ \\
\hline$S_{\infty}$ & $0.080 \pm 0.003$ & $0.182 \pm 0.005$ & $0.657 \pm 0.009$ & $0.777 \pm 0.009$ & $1.73 \quad \pm 0.01$ & $10.96 \quad \pm 0.02$ \\
\hline
\end{tabular}



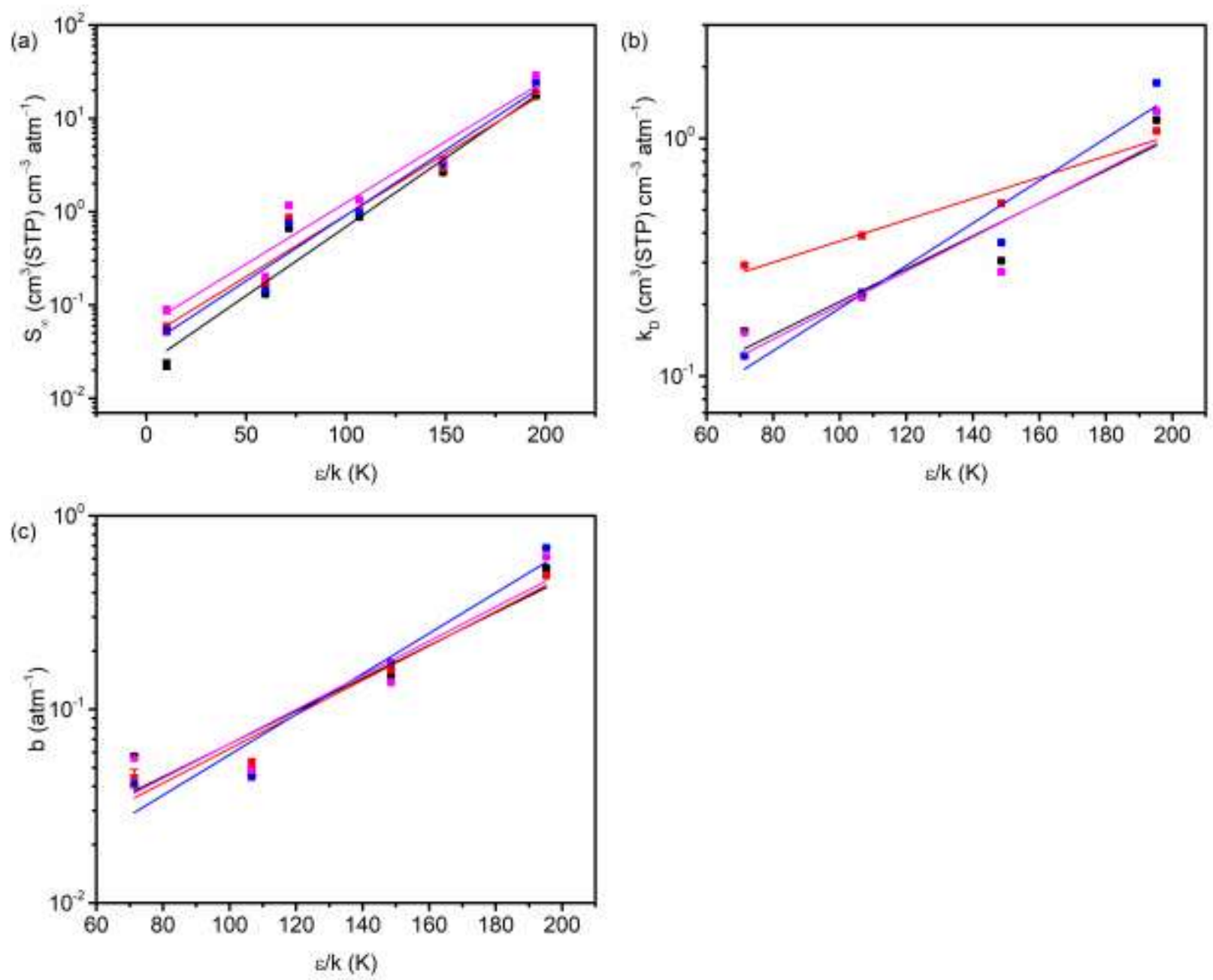

Figure S6: $S_{\infty}, k_{D}$, and $b$ for each polymer with respect to $\varepsilon / k$. The black, red, blue, and pink points represent 6FDA-6HpDA, 6FDA-6FpDA, 6FDA-OHB, and 6FDA-OFB, respectively. 
Table S8: Slopes and intercepts for trends with respect to $\varepsilon / k$ (cf. Equations 15-17 in the main text). $k_{D}$ values are in units of $\mathrm{cm}^{3}(\mathrm{STP}) \mathrm{cm}^{-3} \mathrm{~atm}^{-1}, b$ values are in units of atm ${ }^{-1}$, and $S_{\infty}$ values are in units of $\mathrm{cm}^{3}(\mathrm{STP}) \mathrm{cm}^{-3} \mathrm{~atm}^{-1}$.

\begin{tabular}{|l|l|l|l|c|l|c|}
\hline & \multicolumn{2}{|c|}{$S_{\infty}$} & \multicolumn{2}{c|}{$k_{D}$} & \multicolumn{2}{c|}{$b$} \\
\hline & $A \times 10^{2}$ & $B$ & $C \times 10^{2}$ & $D$ & $E \times 10^{2}$ & $F$ \\
\hline 6FDA-6HpDA & $3.4 \pm 0.2$ & $-3.8 \pm 0.2$ & $1.6 \pm 0.1$ & $-3.2 \pm 0.1$ & $2.0 \pm 0.2$ & $-4.7 \pm 0.3$ \\
\hline 6FDA-6FpDA & $3.0 \pm 0.1$ & $-3.1 \pm 0.2$ & $1.0 \pm 0.1$ & $-2.0 \pm 0.1$ & $2.0 \pm 0.1$ & $-4.8 \pm 0.2$ \\
\hline 6FDA-OHB & $3.3 \pm 0.1$ & $-3.3 \pm 0.2$ & $2.1 \pm 0.1$ & $-3.7 \pm 0.1$ & $2.4 \pm 0.2$ & $-5.2 \pm 0.3$ \\
\hline 6FDA-OFB & $3.0 \pm 0.1$ & $-2.8 \pm 0.2$ & $1.6 \pm 0.1$ & $-3.3 \pm 0.1$ & $2.0 \pm 0.3$ & $-4.7 \pm 0.3$ \\
\hline
\end{tabular}

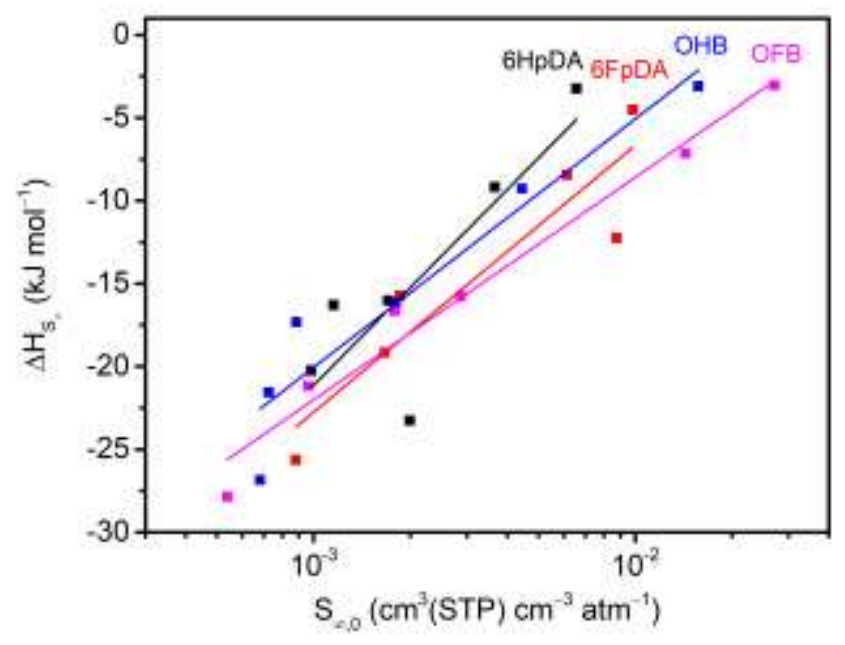

Figure S7: Experimentally derived LFER for $S_{\infty}$ for the four polymers. Each data point represents a specific gas-polymer pair. 

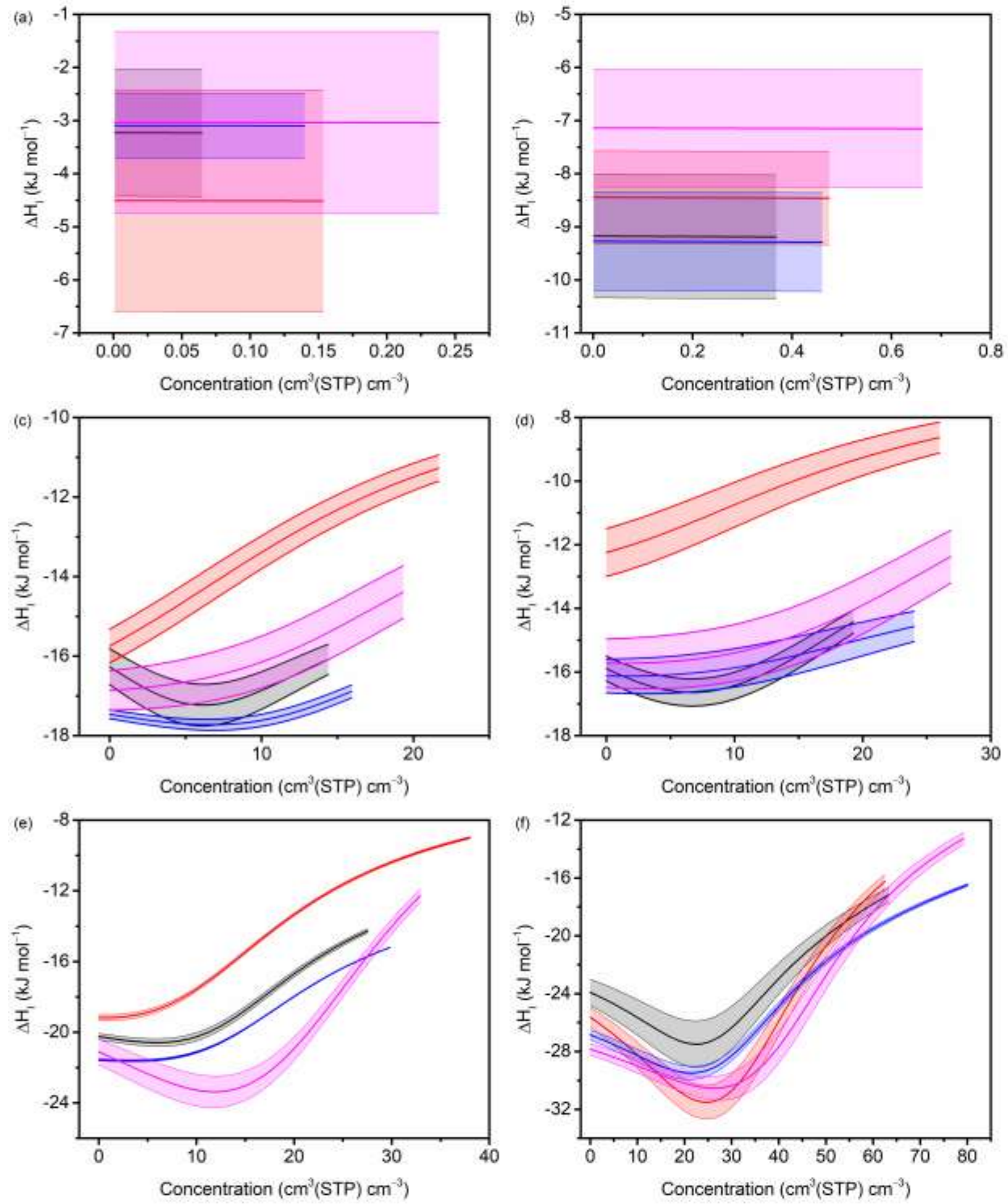

Figure S8: Isosteric heat of sorption for (a) $\mathrm{He}$, (b) $\mathrm{H}_{2}$, (c) $\mathrm{N}_{2}$, (d) $\mathrm{O}_{2}$, (e) $\mathrm{CH}_{4}$, and (f) $\mathrm{CO}_{2}$. The black, red, blue, and pink data sets represent 6FDA-6HpDA, 6FDA-6FpDA, 6FDA-OHB, and 
6FDA-OFB, respectively. The shaded areas represent the error in the calculated isosteric heat of sorption for each gas-polymer pair.
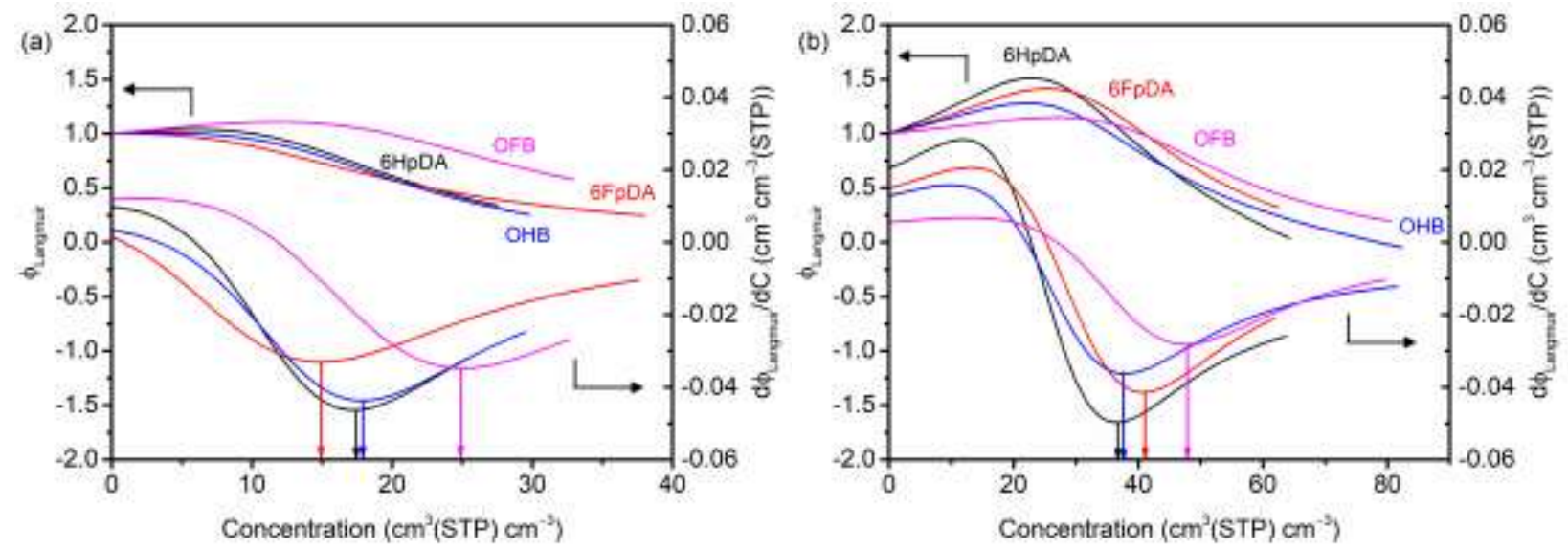

Figure S9: The $\phi_{\text {Langmuir }}$ curves and their derivatives estimated from the calculated isosteric heat of sorption values for (a) $\mathrm{CH}_{4}$ and (b) $\mathrm{CO}_{2}$ for all polymers considered. Downward arrows indicate minima. 